\title{
Two New Resveratrol Tetramers from Upuna borneensis
}

\author{
Tetsuro Ito, ${ }^{*, a}$ Naohito Abe, ${ }^{a}$ Zulfiqar Ali, ${ }^{a}$ Masayoshi Orama, ${ }^{a}$ Toshiyuki Tanaka,,${ }^{b}$ Ryuichi Sawa, ${ }^{c}$ \\ Yoshikazu TaKahashi ${ }^{c}$ Jin Murata, ${ }^{d}$ Dedy Darnaedi,${ }^{e}$ and Munekazu Innuma ${ }^{a}$ \\ ${ }^{a}$ Laboratory of Pharmacognosy, Gifu Pharmaceutical University; ${ }^{b}$ Laboratory of Medicinal Resources, Gifu \\ Pharmaceutical University; 5-6-1 Mitahora-higashi, Gifu 502-8585, Japan: ${ }^{c}$ Microbial Chemistry Research Center; \\ 3-14-23 Kamiosaki, Shinagawa-ku, Tokyo 141-0021, Japan: 'Botanical Gardens, Koishikawa, Graduate School of \\ Science, University of Tokyo; 3-7-1 Hakusan, Bunkyo-ku, Tokyo 112-0001, Japan: and ${ }^{e}$ Indonesian Institute of Sciences; \\ Jalan Ir. H. Juanda 13, Bogor 16122, Indonesia.
}

Received December 15, 2008; accepted February 16, 2009; published online February 25, 2009

\begin{abstract}
Phytochemical investigation of an acetone extract of Upuna borneensis (Dipterocarpaceae) resulted in the isolation of two new resveratrol tetramers, upunaphenols $O$ (1) and $P$ (2). The structures were elucidated by spectroscopic analysis including NMR experiments.
\end{abstract}

Key words Upuna borneensis; Dipterocarpaceae; resveratrol oligomer; upunaphenol

During the course of our research on the isolation and identification of bioactive polyphenols in Dipterocarpaceaeous plants, we have previously reported the structural variety of resveratrol oligomers in Upuna borneensis. ${ }^{1-7)}$ Further detailed examination of the acetone extract of stem yielded two new resveratrol tetramers, upunaphenols $\mathrm{O}(\mathbf{1})$ and $\mathrm{P}(\mathbf{2})$. The structure of isolates $\mathbf{1}$ and $\mathbf{2}$ were elucidated by means of 2D NMR techniques such as ${ }^{1} \mathrm{H}-{ }^{1} \mathrm{H}$ shift correlation spectroscopy (COSY), ${ }^{13} \mathrm{C}-{ }^{1} \mathrm{H}$ COSY, and ${ }^{1} \mathrm{H}-{ }^{13} \mathrm{C}$ heteronuclear multiple-bond correlation (HMBC), and the stereostructures were proposed by analysis of the nuclear Overhauser spectroscopy (NOESY) spectra.

\section{Results and Discussion}

Upunaphenols O (1) $\left([\alpha]_{\mathrm{D}}^{25}+84^{\circ}\right)$, and $\mathrm{P}(\mathbf{2})\left([\alpha]_{\mathrm{D}}^{25}\right.$ $+180^{\circ}$ ) were purified from an acetone-soluble stem segment of $U$. borneensis by column chromatography over silica gel, Sephadex LH-20, octadecyl silica (ODS), and PTLC (preparative TLC).

Upunaphenol O (1), obtained as a pale yellow solid, showed positive reaction to the Gibbs reagent. The molecular formula of $\mathrm{C}_{56} \mathrm{H}_{42} \mathrm{O}_{12}$ was established by an $[\mathrm{M}+\mathrm{H}]^{+}$ion peak at $\mathrm{m} / \mathrm{z} 907.2732$ in the electron spray ionization (ESI)-
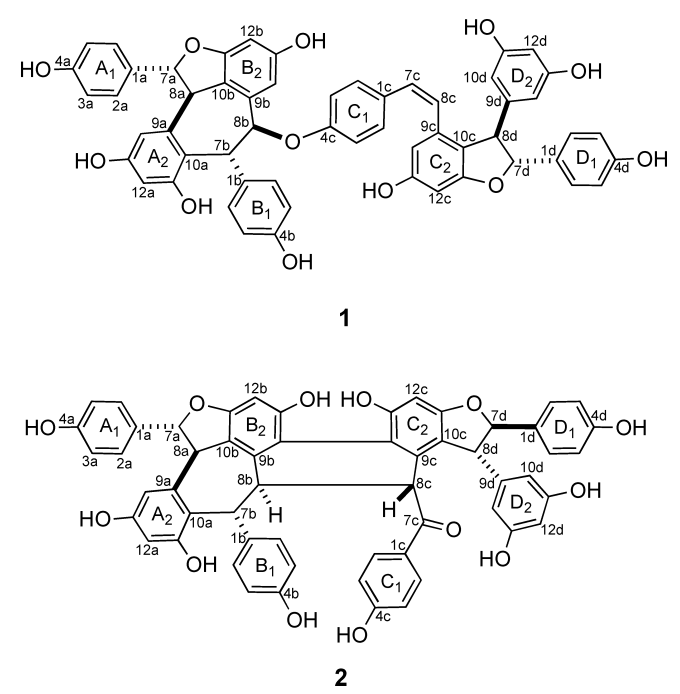

MS together with the NMR spectral data, which suggested that $\mathbf{1}$ is a resveratrol tetramer and bears 36 degrees of unsaturation. Acetylation of $\mathbf{1}$ yielded a nona-acetate (ESI-MS: $\left.\mathrm{m} / \mathrm{z} 1307.3484[\mathrm{M}+\mathrm{Na}]^{+}\right)$, suggesting that $\mathbf{1}$ bears nine phenolic hydroxyl groups. The ${ }^{1} \mathrm{H}-$ and ${ }^{13} \mathrm{C}-\mathrm{NMR}$ spectral data together with ${ }^{1} \mathrm{H}-{ }^{1} \mathrm{H}$ COSY, ${ }^{13} \mathrm{C}-{ }^{1} \mathrm{H}$ COSY and HMBC spectra (Fig. 1, Table 1) showed the presence of ortho-coupled aromatic protons assignable to three 4-hydroxylphenyl groups (rings $\mathrm{A}_{1}, \mathrm{~B}_{1}$ and $\mathrm{D}_{1}$ ), a 4-oxygenated phenyl group (ring $\mathrm{C}_{1}$ ), three sets of meta-coupled aromatic protons on a 1,2,3,5-tetrasubstituted benzene ring (rings $\mathrm{A}_{2}, \mathrm{~B}_{2}$ and $\mathrm{C}_{2}$ ), and a 3,5-dihydroxyphenyl group $\left(\mathrm{D}_{2}\right)$. The NMR spectral data also disclosed the presence of two sets of aliphatic signals characteristic for 2,3-diaryldihydrobenzofuran moieties $(\mathrm{H}-7 \mathrm{a} / \mathrm{H}-8 \mathrm{a} ; \mathrm{H}-7 \mathrm{~d} / \mathrm{H}-8 \mathrm{~d}){ }^{8)}$ two coupled aliphatic methine protons $(\mathrm{H}-7 \mathrm{~b} / \mathrm{H}-8 \mathrm{~b})$, cis-coupled olefinic protons $(\mathrm{H}-7 \mathrm{c} / \mathrm{H}-$ $8 \mathrm{c})$, and nine phenolic hydroxyl groups $\left(\delta_{\mathrm{H}} 7.97-8.64\right)$. Considering the molecular formula, the remaining three oxygens can be allocated to ether linkage. In the HMBC spectrum (Fig. 1), significant ${ }^{3} J$ correlations were observed between $\mathrm{H}-7 \mathrm{a} / \mathrm{C}-2 \mathrm{a}(6 \mathrm{a}), \mathrm{H}-14 \mathrm{a} / \mathrm{C}-8 \mathrm{a}, \mathrm{H}-7 \mathrm{~b} / \mathrm{C}-2 \mathrm{~b}(6 \mathrm{~b}), \mathrm{H}-14 \mathrm{~b} / \mathrm{C}-$ $8 \mathrm{~b}, \mathrm{H}-7 \mathrm{c} / \mathrm{C}-2 \mathrm{c}(6 \mathrm{c}), \mathrm{H}-8 \mathrm{c} / \mathrm{C}-14 \mathrm{c}, \mathrm{H}-7 \mathrm{~d} / \mathrm{C}-2 \mathrm{~d}(6 \mathrm{~d})$, and H-8d/C$10 \mathrm{~d}(14 \mathrm{~d})$, indicating that rings $\mathrm{A}_{1}, \mathrm{~A}_{2}, \mathrm{~B}_{1}, \mathrm{~B}_{2}, \mathrm{C}_{1}, \mathrm{C}_{2}, \mathrm{D}_{1}$, and $\mathrm{D}_{2}$ are attached at C-7a, C-8a, C-7b, C-8b, C-7c, C-8c, C-7d, and $\mathrm{C}-8 \mathrm{~d}$, respectively. Further correlations observed between $\mathrm{H}-8 \mathrm{a} / \mathrm{C}-11 \mathrm{~b}, \mathrm{H}-\mathrm{7b} / \mathrm{C}-11 \mathrm{a}$, and $\mathrm{H}-8 \mathrm{~d} / \mathrm{C}-11 \mathrm{c}$ supported
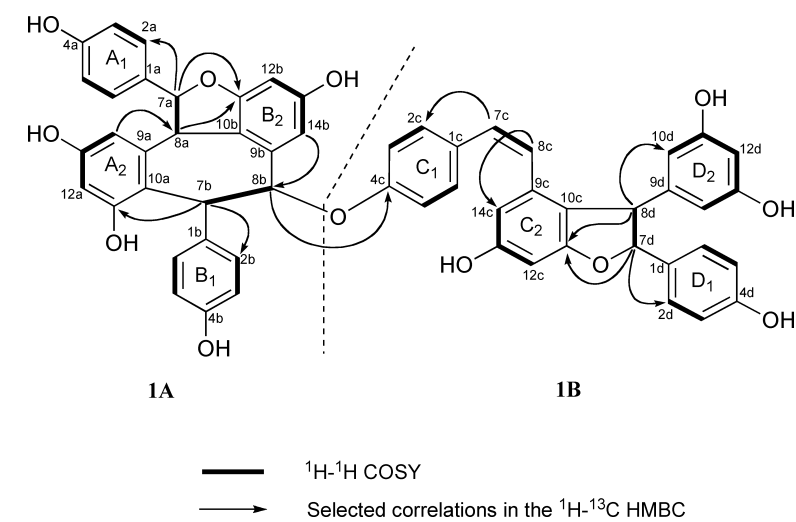

Fig. 1. Selected Correlations in 2D NMR of $\mathbf{1}$ 
Table 1. ${ }^{1} \mathrm{H}$ - and ${ }^{13} \mathrm{C}-\mathrm{NMR}$ Spectral Data of $\mathbf{1}$ and $\mathbf{2}$

\begin{tabular}{|c|c|c|c|c|}
\hline \multirow{2}{*}{ Position } & \multicolumn{2}{|l|}{1} & \multicolumn{2}{|l|}{2} \\
\hline & $\delta_{\mathrm{H}}$ & $\delta_{\mathrm{C}}$ & $\delta_{\mathrm{H}}$ & $\delta_{\mathrm{C}}$ \\
\hline 1a & & 133.5 & & 133.8 \\
\hline $2 a(6 a)$ & $7.42(\mathrm{~d}, 8.4)$ & 130.4 & $7.24(\mathrm{~d}, 8.4)$ & 128.2 \\
\hline $3 a(5 a)$ & $6.94(\mathrm{~d}, 8.4)$ & 116.5 & $6.78(\mathrm{~d}, 8.4)$ & $116.1^{f)}$ \\
\hline $4 a$ & & $158.49^{i)}$ & & $158.1^{g)}$ \\
\hline $7 \mathrm{a}$ & $5.78(\mathrm{~d}, 9.5)$ & 93.5 & $6.10(\mathrm{~d}, 4.2)$ & 90.0 \\
\hline $8 \mathrm{a}$ & $5.32(\mathrm{~d}, 9.5)$ & 52.1 & $4.78(\mathrm{~d}, 4.2)$ & 52.0 \\
\hline $9 \mathrm{a}$ & & 141.4 & & 142.1 \\
\hline $10 \mathrm{a}$ & & 117.9 & & 121.1 \\
\hline $11 \mathrm{a}$ & & $158.1^{c)}$ & 7.93 (brs) & 158.5 \\
\hline $12 \mathrm{a}$ & $6.27(\mathrm{brs})^{a)}$ & 102.1 & $6.06(\mathrm{~d}, 2.2)$ & 102.4 \\
\hline $13 a$ & & 156.8 & & $156.4^{h)}$ \\
\hline $14 \mathrm{a}$ & $6.27(\mathrm{brs})^{a)}$ & 106.6 & $6.55(\mathrm{brs})$ & 105.2 \\
\hline $1 b$ & & 134.0 & & 137.6 \\
\hline $2 b(6 b)$ & $6.82(\mathrm{~d}, 8.6)$ & $130.7^{d)}$ & $6.89(\mathrm{~d}, 8.4)$ & 131.2 \\
\hline $3 b(5 b)$ & $6.52(\mathrm{~d}, 8.6)$ & 115.1 & $6.67(\mathrm{~d}, 8.4)$ & 115.4 \\
\hline $4 \mathrm{~b}$ & & 155.9 & & $156.4^{h)}$ \\
\hline $7 \mathrm{~b}$ & $5.33(\mathrm{~d}, 5.8)$ & 47.1 & $4.04(\mathrm{~d}, 12.8)$ & 45.9 \\
\hline $8 \mathrm{~b}$ & $5.41(\mathrm{~d}, 5.8)$ & 83.0 & $3.54(\mathrm{~d}, 12.8)$ & 46.4 \\
\hline $9 \mathrm{~b}$ & & 137.4 & & 132.8 \\
\hline $10 \mathrm{~b}$ & & 118.2 & & 124.4 \\
\hline $11 \mathrm{~b}$ & & 160.9 & & 156.9 \\
\hline $12 \mathrm{~b}$ & $6.03(\mathrm{~d}, 2.2)$ & 96.9 & $6.32(\mathrm{~s})$ & 97.2 \\
\hline $13 b$ & & $158.54^{i)}$ & & 152.7 \\
\hline $14 b$ & $5.91(\mathrm{~d}, 2.2)$ & 109.5 & & 115.5 \\
\hline $1 \mathrm{c}$ & & $130.6^{e)}$ & & 127.2 \\
\hline $2 c(6 c)$ & $7.02(\mathrm{~d}, 8.6)$ & $130.6^{e)}$ & $6.56(\mathrm{~d}, 8.6)$ & 131.1 \\
\hline $3 c(5 c)$ & $6.79(\mathrm{~d}, 8.6)$ & 116.8 & $6.39(\mathrm{~d}, 8.6)$ & 114.9 \\
\hline $4 \mathrm{c}$ & & 158.0 & & 161.3 \\
\hline $7 \mathrm{c}$ & $6.21(\mathrm{~d}, 13.2)$ & $130.7^{d)}$ & & 195.0 \\
\hline $8 \mathrm{c}$ & $6.03(\mathrm{~d}, 13.2)$ & 126.5 & $4.48(\mathrm{~s})$ & 46.3 \\
\hline $9 \mathrm{c}$ & & 136.8 & & 133.9 \\
\hline $10 \mathrm{c}$ & & 120.0 & & 122.2 \\
\hline $11 \mathrm{c}$ & & 162.5 & & 160.6 \\
\hline $12 \mathrm{c}$ & $6.31(\mathrm{~d}, 2.2)$ & 96.7 & $6.54(\mathrm{~s})$ & 98.9 \\
\hline $13 \mathrm{c}$ & & 159.2 & & 155.2 \\
\hline $14 \mathrm{c}$ & $6.25(\mathrm{~d}, 2.2)$ & 108.4 & & 116.8 \\
\hline $1 \mathrm{~d}$ & & 133.7 & & 133.7 \\
\hline $2 \mathrm{~d}(6 \mathrm{~d})$ & $7.11(\mathrm{~d}, 8.4)$ & 127.9 & $7.32(\mathrm{~d}, 8.6)$ & 127.9 \\
\hline $3 \mathrm{~d}(5 \mathrm{~d})$ & $6.84(\mathrm{~d}, 8.4)$ & 116.1 & $6.93(\mathrm{~d}, 8.6)$ & $116.1^{f)}$ \\
\hline $4 \mathrm{~d}(\mathrm{OH})$ & 8.47 (br s) & $158.1^{c)}$ & & $158.1^{g)}$ \\
\hline $7 \mathrm{~d}$ & $5.32(\mathrm{~d}, 5.3)$ & 93.8 & $5.32(\mathrm{~d}, 6.2)$ & 94.3 \\
\hline $8 \mathrm{~d}$ & $4.04(\mathrm{~d}, 5.3)$ & 57.1 & $4.54(\mathrm{~d}, 6.2)$ & 58.4 \\
\hline $9 \mathrm{~d}$ & & 146.8 & & 145.0 \\
\hline $10 d(14 d)$ & $6.05(\mathrm{~d}, 2.2)$ & 106.8 & $5.75(\mathrm{~d}, 2.0)$ & 107.0 \\
\hline $11 d(13 d)$ & & 159.5 & & 159.1 \\
\hline $12 \mathrm{~d}$ & $6.18(\mathrm{t}, 2.2)$ & 101.9 & $5.52(\mathrm{t}, 2.0)$ & 101.5 \\
\hline \multirow[t]{7}{*}{$\mathrm{OH}$ groups } & 8.64 (br s, OH-4a) & & & \\
\hline & 8.03 (br s, OH-11a) & & & \\
\hline & $8.10(\mathrm{brs}, \mathrm{OH}-13 \mathrm{a})^{b)}$ & & & \\
\hline & 7.97 (br s, OH-4b) & & & \\
\hline & $8.10(\text { br s, OH-13b) })^{b)}$ & & & \\
\hline & 8.37 (br s, OH-13c) & & & \\
\hline & $8.13(\mathrm{br} \mathrm{s}, \mathrm{OH}-11 \mathrm{~d}(13 \mathrm{~d}))$ & & & \\
\hline
\end{tabular}

Measured in acetone- $d_{6}$ at $300 \mathrm{MHz}\left({ }^{1} \mathrm{H}-\mathrm{NMR}\right)$ and $\left.75 \mathrm{MHz}\left({ }^{13} \mathrm{C}-\mathrm{NMR}\right) . \quad a-h\right)$ Overlapping. i) Interchangeable.

the links between $\mathrm{C}-8 \mathrm{a} / \mathrm{C}-10 \mathrm{~b}, \mathrm{C}-7 \mathrm{~b} / \mathrm{C}-10 \mathrm{a}$, and $\mathrm{C}-8 \mathrm{~d} / \mathrm{C}-10 \mathrm{c}$, respectively. Additional cross peaks observed between $\mathrm{H}$ $7 \mathrm{a} / \mathrm{C}-11 \mathrm{~b}, \mathrm{H}-8 \mathrm{~b} / \mathrm{C}-4 \mathrm{c}$, and $\mathrm{H}-7 \mathrm{~d} / \mathrm{C}-11 \mathrm{c}$ supported the presence of ether linkage $(\mathrm{C}-7 \mathrm{a} / \mathrm{O} / \mathrm{C}-11 \mathrm{~b}, \mathrm{C}-8 \mathrm{~b} / \mathrm{O} / \mathrm{C}-4 \mathrm{c} \mathrm{C}$ $7 \mathrm{~d} / \mathrm{O} / \mathrm{C}-11 \mathrm{c})$. The planar structure of upunaphenol $\mathrm{O}$, that included two dihydrobenzofuran rings, has been concluded to be $\mathbf{1}$. The stereostructure of $\mathbf{1}$ was determined by analysis of the NOESY spectrum with the assistance of computer-aided

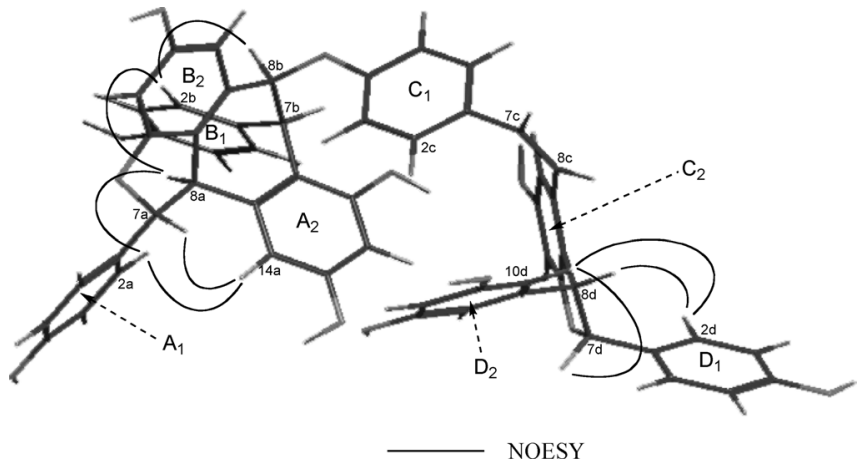

Fig. 2. Selected NOEs Observed in the NOESY Experiment with 1 Model of 1 Drawn by Pcmodel 9.1 Molecular Modeling Program

molecular modeling (Fig. 2). A 3D structure of 1 was generated with the Pcmodel 9.1 molecular modeling program software, using the MMFF94 force field (MM2 type) for energy minimization. ${ }^{9)}$ The trans orientations of $\mathrm{H}-7 \mathrm{a} / \mathrm{H}-8 \mathrm{a}$ and $\mathrm{H}-$ $7 \mathrm{~d} / \mathrm{H}-8 \mathrm{~d}$ on the dihydrobenzofuran rings were confirmed by the distinctive NOEs between H-7a/H-14a, H-14a/H-2a(6a), H-8a/H-2a(6a), H-7d/H-10d(14d), H-2d(6d)/H-10d(14d) and $\mathrm{H}-8 \mathrm{~d} / \mathrm{H}-2 \mathrm{~d}(6 \mathrm{~d})$. The strong NOE $(\mathrm{H}-14 \mathrm{a} / \mathrm{H}-2 \mathrm{a}(6 \mathrm{a}))$ and the large coupling constant $(\mathrm{H}-7 \mathrm{a} / \mathrm{H}-8 \mathrm{a}: J=9.5 \mathrm{~Hz})$ supported the trans diequatrial orientation of two aromatic rings (rings $\mathrm{A}_{1}$ and $\mathrm{A}_{2}$ ) and trans diaxial orientation of $\mathrm{H}-7 \mathrm{a} / \mathrm{H}-8 \mathrm{a}$. Significant NOE between $\mathrm{H}-8 \mathrm{a} / \mathrm{H}-2 \mathrm{~b}(6 \mathrm{~b})$ indicates that ring $\mathrm{B}_{1}$ and $\mathrm{H}-8 \mathrm{a}$ are of $s y n$ orientation. The $s y n$-orientation of $\mathrm{H}-8 \mathrm{~b}$ and ring $B_{1}$ was deduced based on the cross peak for $\mathrm{H}-8 \mathrm{~b} / \mathrm{H}-$ $2 b(6 b)$ in the spectrum. From these results, the relative configuration of six asymmetric carbons in 1, C-7a, C-8a, C-7b, $\mathrm{C}-8 \mathrm{~b}, \mathrm{C}-7 \mathrm{~d}$, and C-8d, were determined as rel- $R, R, R, S, R$, and $R$, respectively. The structure can be regarded as a complex product composed of two resveratrol dimer units $\mathbf{1 A}$ and 1B [unit 1A: resveratrols $A-B$ (resveratrol $A$ : ring $A_{1}-C-7 a-$ C-8a-ring $\mathrm{A}_{2}$ ); unit 1B: resveratrols $\mathrm{C}-\mathrm{D}$ ]. The structures of $\mathbf{1 A}$ and $\mathbf{1 B}$ are identical with resveratrol dimers, ampelopsin A and (Z)- $\varepsilon$-viniferin, respectively. Both compounds have also been isolated from the same material. ${ }^{3)}$ The both dimers are then presumed to be precursors of $\mathbf{1}$.

Upunaphenol P (2) was obtained as a pale yellow amorphous solid. The composition was deduced to be $\mathrm{C}_{56} \mathrm{H}_{40} \mathrm{O}_{13}$ from the pseudo-molecular ion peak of $[\mathrm{M}+\mathrm{H}]^{+}$at $\mathrm{m} / \mathrm{z}$ 921.2527 in the ESI-MS spectrum and the ${ }^{13} \mathrm{C}-\mathrm{NMR}$ spectrum which showed 56 carbon signals. Acetylation of 2 yielded a deca-acetate (ESI-MS: $m / z 1365[\mathrm{M}+\mathrm{Na}]^{+}$), suggesting the presence of 10 phenolic hydroxyl groups. A signal in the ${ }^{13} \mathrm{C}$-NMR spectrum $\left(\delta_{\mathrm{C}} 195.0\right)$ showed the presence of a carbonyl group (C-7c) in the molecule. Analysis of the ${ }^{1} \mathrm{H}$ - and ${ }^{13} \mathrm{C}-\mathrm{NMR}$ spectral data (Table 1 ), ${ }^{1} \mathrm{H}-{ }^{1} \mathrm{H}$, and ${ }^{13} \mathrm{C}-{ }^{1} \mathrm{H}$ COSY and $\mathrm{HMBC}$ spectral data revealed four resveratrol units (resveratrols $\mathrm{A}-\mathrm{D}$ ) consisting of four 4hydroxyphenyl groups (rings $\mathrm{A}_{1}-\mathrm{D}_{1}$ ), a 3,5-dioxygenated1,2-tetrasubstituted benzene ring (ring $\mathrm{A}_{2}$ ), two 3,5-dioxygenated-1,2,6-pentasubstituted benzene rings (rings $\mathrm{B}_{2}$ and $\mathrm{C}_{2}$ ), a 3,5-dihydroxy benzene ring (ring $\mathrm{D}_{2}$ ), two set of mutually coupled aliphatic protons characterized 2,3-diaryldihydrobenzofuran moieties $(\mathrm{H}-7 \mathrm{a} / \mathrm{H}-8 \mathrm{a} ; \mathrm{H}-7 \mathrm{~d} / \mathrm{H}-8 \mathrm{~d})$, and a sequence of three aliphatic protons $(\mathrm{H}-7 \mathrm{~b} / \mathrm{H}-8 \mathrm{~b} / \mathrm{H}-8 \mathrm{c})$. Due to ${ }^{3} J$ cross peaks in the HMBC spectrum (Fig. 3), these partial 


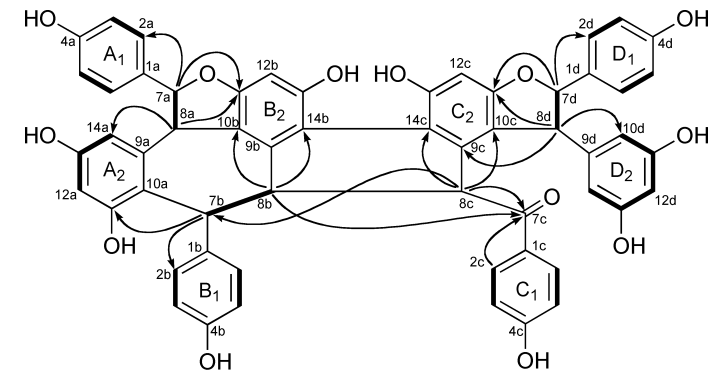

$\longrightarrow \quad{ }^{1} \mathrm{H}-^{-1} \mathrm{H}$ COSY

Fig. 3. Main Connectivities from the HMBC and ${ }^{1} \mathrm{H}-{ }^{1} \mathrm{H}$ COSY Experiments with 2

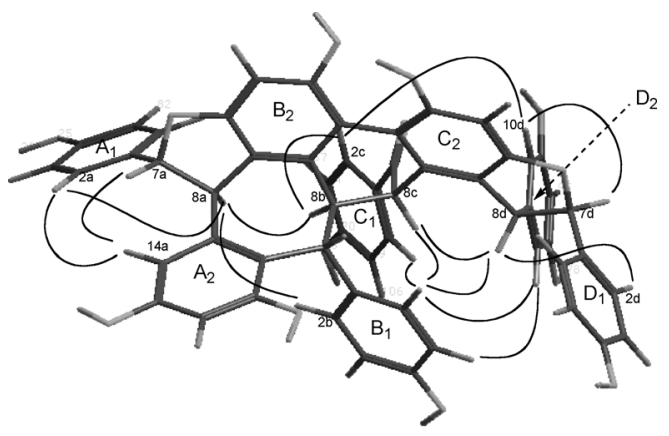

NOESY

Fig. 4. Selected NOEs Observed in the NOESY Experiment with 2 Model of 2 Drawn by Pcmodel 9.1 Molecular Modeling program

structures could be connected except for a bond for C- $14 \mathrm{~b}$ and $\mathrm{C}-14 \mathrm{c}$. The established partial structure satisfied all the oxygen's function and accounted for 36 of the 37 required degrees of unsaturation, which suggested that the ring formation was required. The $\mathrm{C}-14 \mathrm{~b}-\mathrm{C}-14 \mathrm{c}$ bond was assumed after consideration of the molecular skeleton. The planar structure of $\mathbf{2}$ was thus characterized. The planar structure of $\mathbf{2}$ is similar to upunaphenol I except for a presence of a carbonyl group $(\mathrm{C}-7 \mathrm{c})$ instead of an absence of a $\mathrm{C}-\mathrm{C}$ bond $(\mathrm{C}-7 \mathrm{c} / \mathrm{C}-$ 10d) in upunaphenol I. ${ }^{5)}$ The relative configuration of 2 was determined by NOESY experiments (Fig. 4). All trans orientations of two 2,3-diaryldihydrobenzofuran rings were determined by the NOEs observed between $\mathrm{H}-7 \mathrm{a} / \mathrm{H}-14 \mathrm{a}, \mathrm{H}-$ $14 \mathrm{a} / \mathrm{H}-2 \mathrm{a}(6 \mathrm{a}), \quad \mathrm{H}-8 \mathrm{a} / \mathrm{H}-2 \mathrm{~b}(6 \mathrm{~b}), \quad \mathrm{H}-7 \mathrm{~d} / \mathrm{H}-10 \mathrm{~d}(14 \mathrm{~d})$, and $\mathrm{H}-$ $8 \mathrm{~d} / \mathrm{H}-2 \mathrm{~d}(6 \mathrm{~d})$. The relative cis disposition for $\mathrm{H}-8 \mathrm{a}$, ring $\mathrm{B}_{1}$, $\mathrm{H}-8 \mathrm{~b}$, ring $\mathrm{D}_{2}$, and a 4-hydroxybenzoyl group (ring $\mathrm{C}_{1}$ ) were inferred from NOEs for H-8a/H-2a(6a), H-8a/H-8b, H-8b/H$2 \mathrm{c}(6 \mathrm{c})$, and $\mathrm{H}-2 \mathrm{c}(6 \mathrm{c}) / \mathrm{H}-10 \mathrm{~d}(14 \mathrm{~d})$. The results explains well the stereostructure of $\mathbf{2}$, where all the asymmetric carbons are situated in the same manner as upunaphenol I. ${ }^{5)}$ Previously, we have reported the co-presence of resveratrol tetramers, upunaphenols B and $\mathrm{H}-\mathrm{J},(-)$-hopeaphenol and stenophyllol A, in the same material, that could be derived from the main component, (-)-hopeaphenol. ${ }^{3)}$ Compound $\mathbf{2}$ is also presumed to have biogenetic relation with hopeaphenol.

\section{Experimental}

The following instruments were used: optical rotations, JASCO P-1020 polarimeter; UV spectra, Shimadzu UV-2200 spectrophotometer (in $\mathrm{MeOH}$ solution); ${ }^{1} \mathrm{H}$ - and ${ }^{13} \mathrm{C}-\mathrm{NMR}$ spectra, JEOL JNM LA-300 (chemical shift values are presented as $\delta$ values with tetramethylsilane (TMS) as internal standard); ESI-MS, Thermo Fisher Scientific LTQ Orbitrap instrument.

The following adsorbents were used for purification: analytical TLC, Merck Kieselgel $60 \mathrm{~F}_{254}(0.25 \mathrm{~mm})$; preparative TLC, Merck Kieselgel 60 $\mathrm{F}_{254}(0.5 \mathrm{~mm})$; column chromatography, Merck Kieselgel 60, Pharmacia Fine Chemicals AB Sephadex LH-20 and Fuji Silysia Chemical Chromatorex.

Upuna borneensis Sym. was cultivated in Bogor Botanical Garden, Bogor, Indonesia, and its stems were collected in May 2000 and identified by one of co-authors (D.D.). A voucher specimen (number DP-012) has been deposited in Gifu Pharmaceutical University.

Extraction and Isolation of Compounds ( 1 and 2 ): The extraction procedure was the same as that on our previous reports. ${ }^{1-7)} \mathrm{Fr} .7\left(\mathrm{CHCl}_{3}-\mathrm{MeOH}\right.$, 9:1) was further subjected to Sephadex LH-20 CC (MeOH) to give eight fractions (Fr. 7a-Fr. 7h). Compounds $2(17 \mathrm{mg})$ was purified from the fraction of Fr. 7e after purification by Sephadex LH-20 CC (MeOH) and PTLC (EtOAc- $\left.\mathrm{CHCl}_{3}-\mathrm{MeOH}-\mathrm{H}_{2} \mathrm{O}, 15: 8: 4: 1\right)$. Fr. $8\left(\mathrm{CHCl}_{3}-\mathrm{MeOH}, 8: 1\right)$ was divided into seven parts (Fr. 8a-Fr. 8g) in the same way as that of Fr. 7. Sub fraction of Fr. 8f gave $1(12 \mathrm{mg})$ after CC over ODS (MeOH- $\left.\mathrm{H}_{2} \mathrm{O}, 6: 4\right)$.

Compound 1 (Upunaphenol O): A pale yellow solid; $[\alpha]_{\mathrm{D}}^{25}+84^{\circ}(c=0.1$, $\mathrm{MeOH}) ; \mathrm{UV}(\mathrm{MeOH}) \lambda_{\max }(\log \varepsilon): 207$ (4.91), 225sh (4.75), $285(4.07) \mathrm{nm}$; positive ion ESI-MS $m / z$ : $907.2732[\mathrm{M}+\mathrm{H}]^{+}$(Calcd for $\mathrm{C}_{56} \mathrm{H}_{43} \mathrm{O}_{12}$ : 907.2749); ${ }^{1} \mathrm{H}$ - and ${ }^{13} \mathrm{C}-\mathrm{NMR}$ spectral data $\left[{ }^{1} \mathrm{H}(300 \mathrm{MHz}),{ }^{13} \mathrm{C}(75 \mathrm{MHz})\right.$, acetone- $\left.d_{6}\right]$, see Table 1; HMBC correlations, see Fig. 1 (selected) and $\mathrm{H}-$ 2a(6a)/C-4a, C-7a; H-3a(5a)/C-1a, C-4a; OH-4a/C-3a(5a), C-4a; H-7a/C-9a; H-8a/C-1a, C-9a, C-10b; OH-11a/C-10a, C-11a, C-12a; H-12a/C-10a, C11a, C-13a; OH-13a/C-12a, C-13a, C-14a; H-14a/C-10a, C-12a, C-13a; H$2 \mathrm{~b}(6 \mathrm{~b}) / \mathrm{C}-4 \mathrm{~b}, \mathrm{C}-7 \mathrm{~b} ; \mathrm{H}-3 \mathrm{~b}(5 \mathrm{~b}) / \mathrm{C}-1 \mathrm{~b}, \mathrm{C}-4 \mathrm{~b} ; \mathrm{OH}-4 \mathrm{~b} / \mathrm{C}-3 \mathrm{~b}(5 \mathrm{~b}), \mathrm{C}-4 \mathrm{~b} ; \mathrm{H}-7 \mathrm{~b} / \mathrm{C}-$ 9a, C-10a, C-1b, C-9b; H-8b/C-10a, C-1b, C-9b, C-10b, C-14b; H-12b/C10b, C-11b, C-13b, C-14b; OH-13b/C-12b, C-13b, C-14b; H-14b/C-10b, C12b, C-13b; H-2c/C-4c, C-7c; H-3c(5c)/C-1c, C-4c; H-7c/C-9c; H- $8 \mathrm{c} / \mathrm{C}-1 \mathrm{c}$, C-9c, C-10c; H-12c/C-10c, C-11c, C-13c, C-14c; OH-13c/C-12c, C-13c, C$14 \mathrm{c} ; \mathrm{H}-14 \mathrm{c} / \mathrm{C}-10 \mathrm{c}, \mathrm{C}-12 \mathrm{c} ; \mathrm{H}-2 \mathrm{~d}(6 \mathrm{~d}) / \mathrm{C}-4 \mathrm{~d}, \mathrm{C}-7 \mathrm{~d} ; \mathrm{H}-3 \mathrm{~d}(5 \mathrm{~d}) / \mathrm{C}-1 \mathrm{~d}, \mathrm{C}-4 \mathrm{~d} ; \mathrm{OH}-$ 4d/C-3d(5d), C-4d; H-7d/C-9d; H-8d/C-9c, C-10c, C-1d; H-10d(14d)/C$11 \mathrm{~d}(13 \mathrm{~d}), \mathrm{C}-12 \mathrm{~d}$; OH-11d(13d)/C-10d(14d), C-11d(13d), C-12d; H-12d/C$11 \mathrm{~d}(13 \mathrm{~d})$; NOESY correlations: see Fig. 2 (selected) and $\mathrm{H}-3 \mathrm{a}(5 \mathrm{a}) / \mathrm{OH}-4 \mathrm{a}$; H-2a(6a)/H-7a; OH-11a/H-12a, H-7b; H-12a/OH-13a; OH-13a/H-14a; H2b(6b)/H-7b; H-3b(5b)/OH-4b; H-8b/H-3c(5c); H-12b/OH-13b; OH-13b/H$14 \mathrm{~b}, \mathrm{H}-3 \mathrm{c}(5 \mathrm{c}) ; \mathrm{H}-2 \mathrm{c}(6 \mathrm{c}) / \mathrm{H}-10 \mathrm{~d}(14 \mathrm{~d}), \mathrm{OH}-13 \mathrm{c} ; \mathrm{H}-8 \mathrm{c} / \mathrm{H}-8 \mathrm{~d} ; \mathrm{H}-12 \mathrm{c} / \mathrm{OH}-13 \mathrm{c}$; $\mathrm{OH}-13 \mathrm{c} / \mathrm{H}-14 \mathrm{c} ; \mathrm{H}-2 \mathrm{~d}(6 \mathrm{~d}) / \mathrm{H}-7 \mathrm{~d} ; \mathrm{H}-3 \mathrm{~d}(5 \mathrm{~d}) / \mathrm{OH}-4 \mathrm{~d} ; \mathrm{H}-8 \mathrm{~d} / \mathrm{H}-10 \mathrm{~d}(14 \mathrm{~d}) ; \mathrm{H}-$ $10 \mathrm{~d}(14 \mathrm{~d}) / \mathrm{H}-2 \mathrm{c}(6 \mathrm{c}), \mathrm{OH}-11 \mathrm{~d}(13 \mathrm{~d}) ; \mathrm{OH}-11 \mathrm{~d}(13 \mathrm{~d}) / \mathrm{H}-12 \mathrm{~d}$.

Acetylation of 1 A solution of $\mathbf{1}(1 \mathrm{mg})$ in pyridine $(0.5 \mathrm{ml})$ containing $\mathrm{Ac}_{2} \mathrm{O}(0.1 \mathrm{ml})$ was kept at $\mathrm{rt}$ for $24 \mathrm{~h}$. Workup in the usual manner and the purification of the resulting crude product $(1 \mathrm{mg})$ by prep. TLC (nhexane/EtOAc $1: 1)$ afforded a nona-acetate $(1 \mathrm{mg})$. A pale yellow solid; positive ion ESI-MS m/z: $1307.3484[\mathrm{M}+\mathrm{Na}]^{+}$(Calcd for $\mathrm{C}_{74} \mathrm{H}_{60} \mathrm{O}_{21} \mathrm{Na}$ : 1307.3519).

Compound 2 (Upunaphenol P): A pale yellow amorphous solid; $[\alpha]_{\mathrm{D}}^{25}$ $+180^{\circ}(c=0.3, \mathrm{MeOH}) ; \mathrm{UV}(\mathrm{MeOH}) \lambda_{\max }(\log \varepsilon): 225 \mathrm{sh}(4.50), 279(3.96)$, $323(3.52) \mathrm{nm}$; positive ion ESI-MS $\mathrm{m} / \mathrm{z}: 921.2527[\mathrm{M}+\mathrm{H}]^{+}$(Calcd for $\left.\mathrm{C}_{56} \mathrm{H}_{41} \mathrm{O}_{13}: 921.2542\right) ;{ }^{1} \mathrm{H}$ - and ${ }^{13} \mathrm{C}-\mathrm{NMR}$ spectral data $\left[{ }^{1} \mathrm{H}(300 \mathrm{MHz}),{ }^{13} \mathrm{C}\right.$ $\left(75 \mathrm{MHz}\right.$ ), acetone- $\left.d_{6}\right]$, see Table 1; HMBC correlations: see Fig. 3 (selected) and H-2a(6a)/C-4a, C-7a; H-3a(5a)/C-1a, C-4a; H-7a/C-1a, C-9a; H8a/C-10a, C-10b; OH-11a/C-10a, C-11a; H-12a/C-10a, C-13a, C-14a; H14a/C-10a, C-13a; H-2b(6b)/C-4b, C-7b ; H-3b(5b)/C-1b, C-4b; H-7b/C-9a, C-10a, C-1b; H-8b/C-1b, C-9b; H-12b/C-10b, C-11b, C-13b, C-14b; H$2 \mathrm{c}(6 \mathrm{c}) / \mathrm{C}-4 \mathrm{c} ; \mathrm{H}-3 \mathrm{c}(5 \mathrm{c}) / \mathrm{C}-1 \mathrm{c}, \mathrm{C}-4 \mathrm{c} ; \mathrm{H}-8 \mathrm{c} / \mathrm{C}-9 \mathrm{c} ; \mathrm{H}-12 \mathrm{c} / \mathrm{C}-10 \mathrm{c}, \mathrm{C}-11 \mathrm{c}, \mathrm{C}-13 \mathrm{c}$, C-14c; H-2d(6d)/C-4d, C-7d; H-3d(5d)/C-1d, C-4d; H-7d/C-1d, C-9d; H8d/C-1d; H-10d(14d)/C-11d(13d), C-12d; NOESY correlations: see Fig. 4 (selected) and H-2a(6a)/H-7a; OH-11a/H-12a, H-7b; H-2b(6b)/H-7b; H$3 \mathrm{c}(5 \mathrm{c}) / \mathrm{H}-10 \mathrm{~d}(14 \mathrm{~d}) ; \mathrm{H}-2 \mathrm{~d}(6 \mathrm{~d}) / \mathrm{H}-7 \mathrm{~d} ; \mathrm{H}-8 \mathrm{~d} / \mathrm{H}-10 \mathrm{~d}(14 \mathrm{~d})$.

Acetylation of 2 Compound $\mathbf{2}(2 \mathrm{mg})$ was treated in the same way as $\mathbf{1}$ to afford a deca-acetate $(2 \mathrm{mg})$. A pale yellow solid; positive ion ESI-MS $m / z: 1363.3398[\mathrm{M}+\mathrm{Na}]^{+}\left(\mathrm{Calcd}\right.$ for $\mathrm{C}_{76} \mathrm{H}_{60} \mathrm{O}_{23} \mathrm{Na}$ : 1363.3418).

\section{References and Notes}

1) Ito T., Tanaka, T., Ali Z., Takahashi Y., Sawa R., Nakaya K., Murata J., Darnaedi D., Iinuma M., Heterocycles, 63, 129-136 (2004).

2) Ali Z., Ito T., Tanaka T., Nakaya K., Murata J., Darnaedi D., Inuma M., Phytochemistry, 65, 2141-2146 (2004).

3) Ito T., Furusawa M., Tanaka T., Ali Z., Iliya I., Nakaya K., Murata J., Darnaedi D., Iinuma M., Chem. Pharm. Bull., 53, 219-224 (2005).

4) Ito T., Iliya I., Tanaka T., Nakaya K., Akao, Y., Nozawa, Y., Murata J., Darnaedi D., Iinuma M., Heterocycles, 65, 173-179 (2005). 
5) Ito T., Ali Z., Furusawa M., Iliya I., Tanaka T., Nakaya K., Murata J., Darnaedi D., Oyama M., Iinuma M., Chemistry \& Biodiversity, 2, 1673-1684 (2005).

6) Ito T., Ali Z., Iliya I., Furusawa M., Tanaka T., Nakaya K., Takahashi Y., Sawa R., Murata J., Darnaedi D., Iinuma M., Helv. Chim. Acta, 88, 23-34 (2005).

7) Ito T., Ali Z., Furusawa M., Iliya I., Tanaka T., Nakaya K., Murata J.,
Darnaedi D., Iinuma M., Chem. Pharm. Bull., 54, 363-367 (2006).

8) Seo E.-K., Chai H., Constant H. L., Santisul T., Reutrakul V., Beecher C. W. W., Farnsworth N. R., Cordell G. A., Pezzuto J. M., Kinghorn A. D., J. Org. Chem., 64, 6976-6983 (1999).

9) PCMODEL v 9.0, Serena Software, Box 3076, Bloomington, IN 47402-3076. 